\title{
Fast Computing of Discrete Cosine and Sine Transforms of Types VI and VII
}

\author{
Ravi K. Chivukula and Yuriy A. Reznik \\ Qualcomm Inc., 5775 Morehouse Dr., San Diego, CA 92122, USA
}

\begin{abstract}
We propose fast algorithms for computing Discrete Sine and Discrete Cosine Transforms (DCT and DST) of types VI and VII. Particular attention is paid to derivation of fast algorithms for computing DST-VII of lengths 4 and 8 , which are currently under consideration for inclusion in ISO/IEC/ITU-T High Efficiency Video Coding (HEVC) standard.
\end{abstract}

Keywords: Discrete Cosine Transform, Discrete Sine Transform, DCT, DST, DST-VII, factorization, multiplicative complexity, video coding, HEVC.

\section{INTRODUCTION}

The Discrete Sine and Cosine Transforms (DST and DCT) have already received considerable attention due to their use in source coding. The DCT of type-II (DCT-II) is at the core of standards for image and video compression, such as JPEG, H.26x-series, and MPEG 1-4 standards. ${ }^{1}$ The DST of type-I was suggested as a basis for Recursive Block Coding technique. ${ }^{2}$ The DCT and DST of type IV are used in design of Lapped Orthogonal Transforms ${ }^{3}$ serving as core filterbanks in speech and audio coding algorithms, such as G.722.1, G.718, MPEG-4 AAC, and others. ${ }^{4}$

The DCT and DST transforms of types I, II, III, and IV, form a group of so-called "even" sinusoidal transforms. $^{5-7}$ Such transforms are very well studied, and a number of efficient technique exists for their computation. ${ }^{1,8-14}$ Design of integer approximations of such transforms was also a subject of active study in recent years. ${ }^{8,15,16}$

Much less known is a group of so-called "odd" sinusoidal transforms: DST and DCT of types V, VI, VII, and VIII. Existence of such transforms was discovered by A. Jain in $1979 .{ }^{5}$ A complete set of such transforms was defined later by Wang and Hunt. ${ }^{6}$ However, not much work has followed. Surveys of several such related results can be found in. ${ }^{7,8}$ To the best of our knowledge, no fast algorithms were specifically designed for computing of such transforms.

Our interest in transforms of types VI and VII is based on new applications of DST-VI/VII transforms in image/video coding. Recently, Han, Saxena, and Rose ${ }^{17}$ have shown that Karhunen-Loeve Transform (KLT) for residual signals, such as ones produced by Intra-Prediction process in video coding, ${ }^{18}$ can be well approximated by DST-VII. This was further validated in ongoing experimental work on High Efficiency Video Coding (HEVC) standard, ${ }^{19}$ resulting in inclusion of DST-VI/VII transforms in the Working Draft of this standard. ${ }^{20,21}$ The existence of fast algorithms for computing of such transforms can provide additional argument for using these transforms in HEVC and future image and video coding standards.

This paper is organized as follows. In Section 2, we offer definitions of DCT and DST transforms of even and odd types, and survey several known results. In Section 3, we derive mappings between DST of types VI/VII and the DFT. In Section 4, we show how this mapping to DFT can be used to design algorithms for computing DST-VI/VII transforms of lengths $N=4,8$. Finally, in Section 5, we analyze complexity of the proposed algorithms.

Further author information: (Send correspondence to Yuriy A. Reznik)

Ravi K. Chivukula: E-mail: rchivuku@qualcomm.com, Telephone: 19282745288

Yuriy A. Reznik: E-mail: yreznik@ieee.org, Telephone: 12062650309. 


\section{DEFINITIONS AND SOME KNOWN FACTS}

Hereafter, by letters $N$ or $M$ we will denote transform sizes, by $\Re($.$) and \Im($.$) we will denote real and imaginary$ parts of complex numbers, and by $j=\sqrt{-1}$ we will denote imaginary unit.

The matrix of an $N$-point Discrete Fourier Transform (DFT) will be defined as follows

$$
\text { DFT: } \quad\left[F_{N}\right]_{m n}=\frac{1}{\sqrt{N}} e^{-j \frac{2 \pi m n}{N}}, m, n=0, \ldots, N-1 .
$$

The matrices of $M$-point DCT and DST transforms of types II and III will be defined as

$$
\begin{array}{lrrr}
\text { DCT-II: } & {\left[C_{M}^{I I}\right]_{m n}=\sqrt{\frac{2}{M}} \kappa_{m} \cos \left(\frac{m(2 n+1) \pi}{2 M}\right),} & m, n=0, \ldots, M-1 ; \\
\text { DCT-III: } \quad\left[C_{M}^{I I I}\right]_{m n}=\sqrt{\frac{2}{M}} \kappa_{n} \cos \left(\frac{(2 m+1) n \pi}{2 M}\right), & m, n=0, \ldots, M-1 ; \\
\text { DST-II: } & {\left[S_{M}^{I I}\right]_{m n}=\sqrt{\frac{2}{M}} \kappa_{m} \sin \left(\frac{m(2 n-1) \pi}{2 M}\right),} & m, n=1, \ldots, M ; \\
\text { DST-III: } & {\left[S_{M}^{I I I}\right]_{m n}=\sqrt{\frac{2}{M}} \kappa_{n} \sin \left(\frac{(2 m-1) n \pi}{2 M}\right),} & m, n=1, \ldots, M ;
\end{array}
$$

The matrices of DCT and DST transforms of types IV and VII (also known as "odd" transforms of types II and III ${ }^{6,7}$ ) will be defined as

$$
\begin{array}{llll}
\text { DCT-VI: } & {\left[C_{M}^{V I}\right]_{m n}=\frac{2}{\sqrt{2 M-1}} \kappa_{m} \lambda_{n} \cos \left(\frac{m(2 n+1) \pi}{2 M-1}\right),} & m, n=0, \ldots, M-1 ; \\
\text { DCT-VII: } & {\left[C_{M}^{V I I}\right]_{m n}=\frac{2}{\sqrt{2 M-1}} \lambda_{m} \kappa_{n} \cos \left(\frac{(2 m+1) n \pi}{2 M-1}\right),} & m, n=0, \ldots, M-1 ; \\
\text { DST-VI: } & {\left[S_{M-1}^{V I}\right]_{m n}=\frac{2}{\sqrt{2 M-1}} \sin \left(\frac{m(2 n-1) \pi}{2 M-1}\right),} & m, n=1, \ldots, M-1 ; \\
\text { DST-VII: } & {\left[S_{M-1}^{V I I}\right]_{m n}=\frac{2}{\sqrt{2 M-1}} \sin \left(\frac{(2 m-1) n \pi}{2 M-1}\right),} & m, n=1, \ldots, M-1 ;
\end{array}
$$

In above formulae, normalization constants $\kappa_{i}$ and $\lambda_{i}$ are defined as follows

$$
\kappa_{i}=\left[\begin{array}{ll}
\frac{1}{\sqrt{2}}, & \text { if } i=0 \text { or } i=M \\
1, & \text { otherwise }
\end{array}, \quad \lambda_{i}=\left[\begin{array}{ll}
\frac{1}{\sqrt{2}}, & \text { if } i=M-1 \\
1, & \text { otherwise }
\end{array} .\right.\right.
$$

All these definitions are standard, see, e.g. Britanak, Rao, and Yip. ${ }^{8}$ We further follow convention of Wang and Hunt $^{6}$ that associate DCT-VI/VII transforms of length $M$ with DST-VI/VII transforms of lengths $M-1$. We will explain the nature of this association in the next section.

The following relationships between these transforms are well known: ${ }^{8}$

$$
\left(C_{N}^{I I}\right)^{-1}=C_{N}^{I I I} ; \quad\left(S_{N}^{I I}\right)^{-1}=S_{N}^{I I I} ; \quad\left(C_{N+1}^{V I}\right)^{-1}=C_{N+1}^{V I I} ; \quad\left(S_{N}^{V I}\right)^{-1}=S_{N}^{V I I} .
$$

\subsection{Connection Between DCT and DST of Even and Odd Types and the DFT}

We now retrieve two known mappings between DCT and DST of even and odd types and the DFT. ${ }^{6}$

Proposition 1. The following holds:

$$
\begin{aligned}
\left(\begin{array}{cc}
C_{M}^{I I} & \\
& J_{M} S_{M}^{I I} J_{M}
\end{array}\right) & =A_{2 M}^{I I I} U_{2 M} H_{2 M}^{-1} F_{2 M} A_{2 M}^{I I}, \\
\left(\begin{array}{cc}
C_{M}^{V I} & \\
& J_{M-1} S_{M-1}^{V I} J_{M-1}
\end{array}\right) & =A_{2 M-1}^{I I I} U_{2 M-1} H_{2 M-1}^{-1} F_{2 M-1} A_{2 M-1}^{I I},
\end{aligned}
$$

where $I_{N}$ and $J_{N}$ denote $N \times N$ identity and order-reversal matrices, correspondingly,

$$
A_{2 M}^{I I}=\frac{1}{\sqrt{2}}\left(\begin{array}{cc}
I_{M} & J_{M} \\
J_{M} & -I_{M}
\end{array}\right), \quad A_{2 M-1}^{I I}=\frac{1}{\sqrt{2}}\left(\begin{array}{ccc}
I_{M-1} & & J_{M-1} \\
& \sqrt{2} & \\
J_{M-1} & & -I_{M-1}
\end{array}\right),
$$




$$
\begin{gathered}
A_{2 M}^{I I I}=\frac{1}{\sqrt{2}}\left(\begin{array}{cccc}
\sqrt{2} & & & \\
& I_{M-1} & & -J_{M-1} \\
& & \sqrt{2} & \\
& J_{M-1} & & I_{M-1}
\end{array}\right), A_{2 M-1}^{I I I}=\frac{1}{\sqrt{2}}\left(\begin{array}{ccc}
\sqrt{2} & & \\
& I_{M-1} & -J_{M-1} \\
& J_{M-1} & I_{M-1}
\end{array}\right), \\
H_{2 M}=\left(\begin{array}{cccc}
1 & & & \\
& \frac{1-j}{2} I_{M-1} & & \frac{1+j}{2} J_{M-1} \\
& & 1 & \\
& \frac{1+j}{2} J_{M-1} & & \frac{1-j}{2} I_{M-1}
\end{array}\right), \quad H_{2 M-1}=\left(\begin{array}{ccc}
1 & & \\
& \frac{1-j}{2} I_{M-1} & \frac{1+j}{2} J_{M-1} \\
& \frac{1+j}{2} J_{M-1} & \frac{1-j}{2} I_{M-1}
\end{array}\right),
\end{gathered}
$$

and where

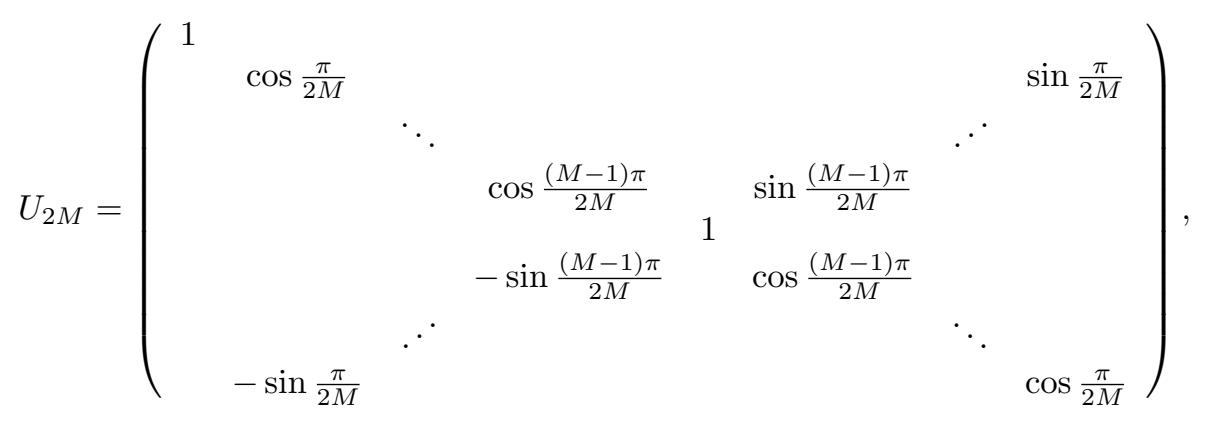

and

$$
U_{2 M-1}=\left(\begin{array}{ccccccc}
1 & & & & & & \\
& \cos \frac{\pi}{2 M-1} & & & & & \sin \frac{\pi}{2 M-1} \\
& & \ddots & & & & \\
& & & \cos \frac{(M-1) \pi}{2 M-1} & \sin \frac{(M-1) \pi}{2 M-1} & & \\
& & & -\sin \frac{(M-1) \pi}{2 M-1} & \cos \frac{(M-1) \pi}{2 M-1} & & \\
& & & & & \ddots & \\
& & & & & & \cos \frac{\pi}{2 M-1}
\end{array}\right) .
$$

Proof. Wang and Hunt ${ }^{6}$ have already established mappings between DST and DCT of types II and IV and so-called discrete $W$ transform of type II (cf. [6, Equations 99,100]):

$$
\left[W_{N}^{I I}\right]_{m, n}=\sqrt{\frac{2}{N}} \sin \left(\frac{\pi}{4}+\frac{m(2 n+1) \pi}{N}\right), \quad m, n=0, \ldots, N-1 .
$$

By further connecting $W_{N}^{I I}$ to DFT [6, Equations 51,56]:

$$
H_{N} U_{N}^{-1} W_{N}^{I I}=F_{N} .
$$

we obtain (2) and (3).

By examining the first expression (2), we note, that a pair of $M$-point type-II DCT/DST transforms becomes connected to DFT of length $2 M$. On the other hand, in the second case (3), the pair of type-VI DCT/DST transforms becomes mapped to DFT of length $2 M-1$. Such mapping involves a $M$-point DCT-VI and an $M$ - 1-point DST-VI. By inverting of both sides in (2) and (3) we can obtain similar relationships between DFT and pairs of DCT/DST transforms of types III and VII. This follows from (1).

We also note that, in principle, formulae (2) and (3) already prove existence of fast algorithms for DCT and DST of all mentioned types. Such algorithms must be exist simply because there exist fast algorithms for DFT, such as Cooley-Tukey FFT, Prime-Factor FFT, Winograd DFT algorithm, and others. ${ }^{22-26}$ On the other hand, such algorithms constructed using mappings (2) and (3) may not be least complex possible. We note, for example, that besides DFT, computation according to (2) or (3) also involves $M-1$ planar rotation operations (given by matrix $U$ ), which adds considerable overhead. 
From literature on DCT and DST transforms of types II and III, ${ }^{1,8-11}$ we know, that there exist several more efficient mappings of such transforms to DFT. ${ }^{1,9}$ As we shall show next, there are also simpler mappings between DFT and DST/DCT transforms of types IV and VII. For simplicity of presentation, we will focus mainly on DST, but same approach can be easily extended to DCT as well.

\section{DIRECT MAPPINGS BETWEEN DST-VI/VII AND DFT}

For convenience, we will now use $N$ instead of $M-1$ to define the size of DST-VI/VII transforms. Our main result establishing direct mapping between such transforms and the DFT is given below.

THEOREM 3.1. The following holds:

$$
S_{N}^{V I I}=\frac{1}{2} R \Im\left[F_{2 N+1}\right] Q P
$$

where $\Im\left[F_{2 N+1}\right]$ denotes imaginary part of DFT of length $2 N+1, R$ is an $N \times(2 N+1)$ matrix collecting odd-indexed outputs:

$$
[R]_{m n}=\left[\begin{array}{lll}
1, & \text { if } n=2 m+1, & m=0, \ldots, N-1 \\
0, & \text { otherwise } & n=0, \ldots, 2 N
\end{array}\right.
$$

$Q$ is an $(2 N+1) \times N$ expansion matrix

$$
Q=\left(\begin{array}{c}
0 \\
-J_{N} \\
I_{N}
\end{array}\right)
$$

and $P$ is a permutation matrix, defined as follows:

$$
[P]_{m n}=\left[\begin{array}{ll}
1, & \text { if } n=2 m, \\
1, & \text { if }\left\lceil\frac{N}{2}\right\rceil+n=2\left\lfloor\frac{N}{2}\right\rfloor-1-2 m, \quad m, n=0, \ldots, N-1 . \\
0, & \text { otherwise }
\end{array}\right.
$$

Proof. Let $x=\left[x_{0}, \ldots, x_{N-1}\right]^{T}$ be real vector representing input signal. By $X=S_{N}^{V I I I} x$ we denote output of DST-VII applied to $x$ :

$$
X_{k}=\frac{1}{\sqrt{2 N+1}} \sum_{n=0}^{N-1} x_{n} \sin \frac{\pi(2 k+1)(n+1)}{2 N+1}, \quad k=0, \ldots, N-1 .
$$

Let us also define an intermediate $2 N+1$-point vector $y$ :

$$
y=Q P x=\left[\begin{array}{rlrl}
y_{0} & =0, & & \\
y_{N+1+n} & =x_{2 n}, & & n=0, \ldots,\left\lceil\frac{N}{2}\right\rceil-1, \\
y_{N+1+\left\lceil\frac{N}{2}\right\rceil+n} & =x_{2\left\lfloor\frac{N}{2}\right\rfloor-1-2 n}, & & n=0, \ldots,\left\lfloor\frac{N}{2}\right\rfloor-1, \\
y_{1+n} & =-y_{2 N-n}, & & n=0, \ldots, N-1,
\end{array}\right.
$$

and apply DFT to it

$$
Y_{k}=\frac{1}{\sqrt{2 N+1}} \sum_{n=0}^{2 N+1} y_{n} e^{-j \frac{2 \pi k n}{2 N+1}} ; \quad k=0, \ldots, 2 N
$$

Now, let's take a look at odd imaginary DFT components $(k=0, \ldots, N-1)$ :

$$
\begin{aligned}
\sqrt{2 N+1} \Im\left[Y_{2 k+1}\right] & =-\sum_{n=1}^{2 N} y_{n} \sin \frac{2 \pi(2 k+1) n}{2 N+1} \\
& =-\sum_{n=1}^{N}\left[y_{n} \sin \frac{2 \pi(2 k+1) n}{2 N+1}+y_{2 N+1-n} \sin \frac{2 \pi(2 k+1)(2 N+1-n)}{2 N+1}\right],
\end{aligned}
$$


From mapping (8) we know that $y_{n}=-y_{2 N+1-n}$. Hence (9) turns into

$$
\begin{aligned}
\sqrt{2 N+1} \Im\left[Y_{2 k+1}\right] & =\sum_{n=1}^{N} y_{2 N+1-n}\left[\sin \frac{2 \pi(2 k+1) n}{2 N+1}-\sin \frac{2 \pi(2 k+1)(2 N+1-n)}{2 N+1}\right] \\
& =2 \sum_{n=1}^{N} y_{2 N+1-n} \sin \frac{\pi(2 k+1)(2 N+1-2 n)}{2 N+1} \\
& =2 \sum_{n=1}^{N} y_{2 N+1-n} \sin \frac{2 \pi(2 k+1) n}{2 N+1},
\end{aligned}
$$

or by using substitution $n^{\prime}=N-n$ in (10):

$$
\sqrt{2 N+1} \Im\left[Y_{2 k+1}\right]=2 \sum_{n=N-1}^{0} y_{N+1+n} \sin \frac{\pi(2 k+1)(2 n+1)}{2 N+1} .
$$

Let's now assume that $N$ is even. Similar argument holds for odd $N$. We write

$$
\begin{aligned}
\frac{\sqrt{2 N+1}}{2} \Im\left[Y_{2 k+1}\right] & =\sum_{n=0}^{\frac{N}{2}-1} y_{N+1+n} \sin \frac{\pi(2 k+1)(2 n+1)}{2 N+1} \\
& +\sum_{n=0}^{\frac{N}{2}-1} y_{\frac{3 N}{2}+1+n} \sin \frac{\pi(2 k+1)(2 n+N+1)}{2 N+1}
\end{aligned}
$$

where, based on (8), the first sum (11) receives quantities $y_{N+1+n}=x_{2 n}$, while the second sum (12) receives $y_{\frac{3 N}{2}+1+n}=x_{N-1-2 n}$.

By putting everything together, we obtain

$$
\begin{aligned}
\frac{\sqrt{2 N+1}}{2} \Im\left[Y_{2 k+1}\right] & =\sum_{n=0}^{\frac{N}{2}-1} x_{2 n} \sin \frac{\pi(2 k+1)(2 n+1)}{2 N+1}+\sum_{n=0}^{\frac{N}{2}-1} x_{N-1-2 n} \sin \frac{\pi(2 k+1)(2 n+N+1)}{2 N+1} \\
& =\sum_{n=0}^{\frac{N}{2}-1} x_{2 n} \sin \frac{\pi(2 k+1)(2 n+1)}{2 N+1}+\sum_{n=0}^{\frac{N}{2}-1} x_{2 n+1} \sin \frac{\pi(2 k+1)((2 n+1)+1)}{2 N+1} \\
& =\sqrt{2 N+1} X_{k} .
\end{aligned}
$$

口

We show the flow-graph of mapping (4) in Figure 1.a. It can be observed that only reordering and negation operations are needed to compute DST-VII via DFT. We next show two simplified mappings between DST-VII and DFT, that can be derived from (4).

COROLLARY 1. The following holds:

$$
\begin{aligned}
& S_{N}^{V I I}=R \Im\left[F_{2 N+1}\right] Z_{0} P, \\
& S_{N}^{V I}=-R \Im\left[F_{2 N+1}\right] Z_{1} P,
\end{aligned}
$$

where matrices $R$ and $P$ are as defined in (5) and (7) correspondingly, and where $Z_{0}$ and $Z_{1}$ are $(2 N+1) \times N$ expansion matrices:

$$
Z_{0}=\left(\begin{array}{c}
0 \\
O_{N} \\
I_{N}
\end{array}\right)
$$




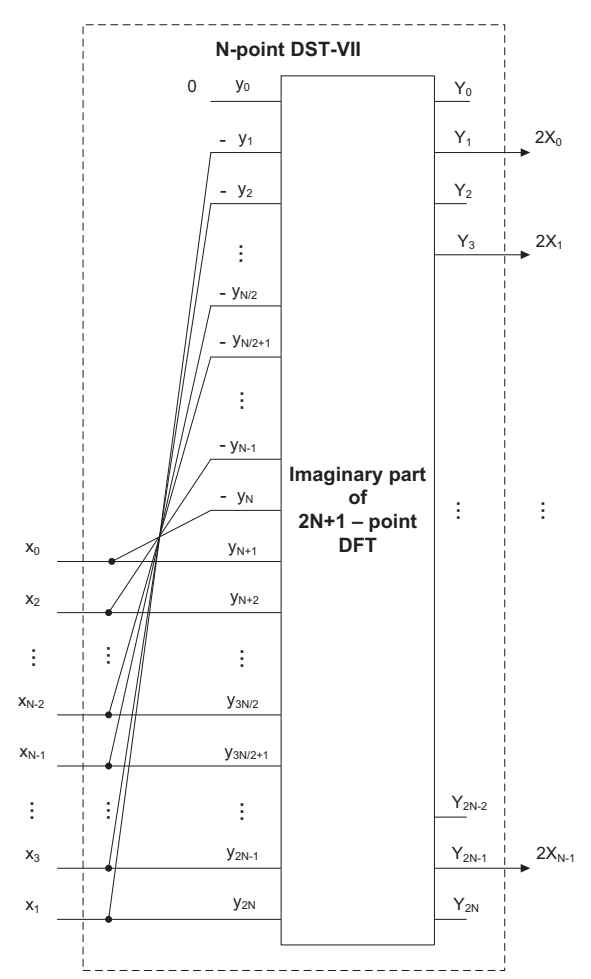

(a)

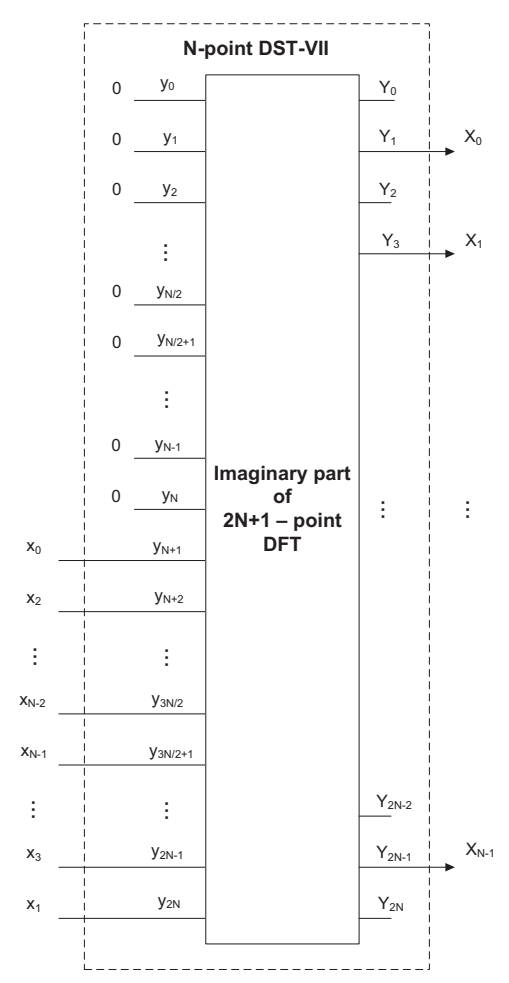

(b)

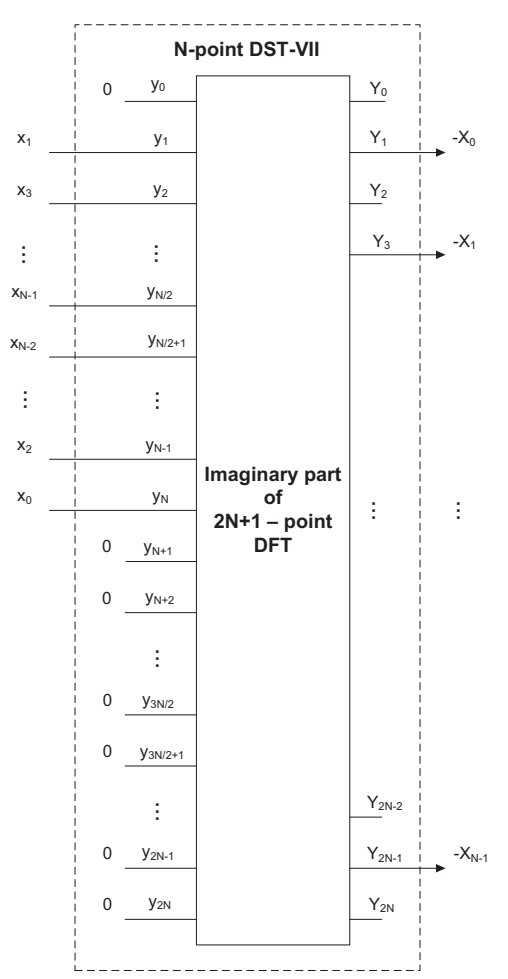

(c)

Figure 1. Flow-graphs of mappings between $N$-point DST-VII and $2 N+1$-point DFT, drawn when $N$ is even. Flow-graph (a) corresponds to mapping (4). Flog-graphs (b) and (c) correspond to simplified mappings (13) and (14) correspondingly.

and

$$
Z_{1}=\left(\begin{array}{c}
0 \\
J_{N} \\
O_{N}
\end{array}\right)
$$

where $O_{N}, I_{N}$, and $J_{N}$, denote $N \times N$ zero, identity, and order-reversal matrices correspondingly.

Proof. This follows by observing that the use of mapping $y_{n}=-y_{2 N+1-n}$ in (9) makes both sums equivalent, which introduces a factor of 2 in (10). Turning either lover or upper part of $y$ to zero would leave only one of those sums, producing same exact result, but without factor of 2 .

We show flow-graphs corresponding to mapping (13) and (14) in Figures 1.a and 1.b correspondingly. It can be observed that DST-VII can be computed by simply producing particularly re-ordered and zero-padded sequence as input to DFT, and collecting imaginary parts of odd-indexed DFT output values. Moreover, in practice, one does not even have to compute full DFT to produce DST-VII output. We will discuss design of such "pruned" DFT in the next section.

\section{FAST ALGORITHMS FOR COMPUTING DST-VI/VII OF LENGTHS N=4,8}

Based on previous discussion, it follows that fast factorization of DST-VII transform can be constructed by:

- Selecting mapping between DST-VII and DFT;

- Selecting fast factorization of DFT of length $2 N+1$;

- Pruning DFT flow-graph, leaving only paths leading to odd-indexed imaginary output values, and

- Connecting those odd-indexed DFT outputs to corresponding output lines of DST-VII. 


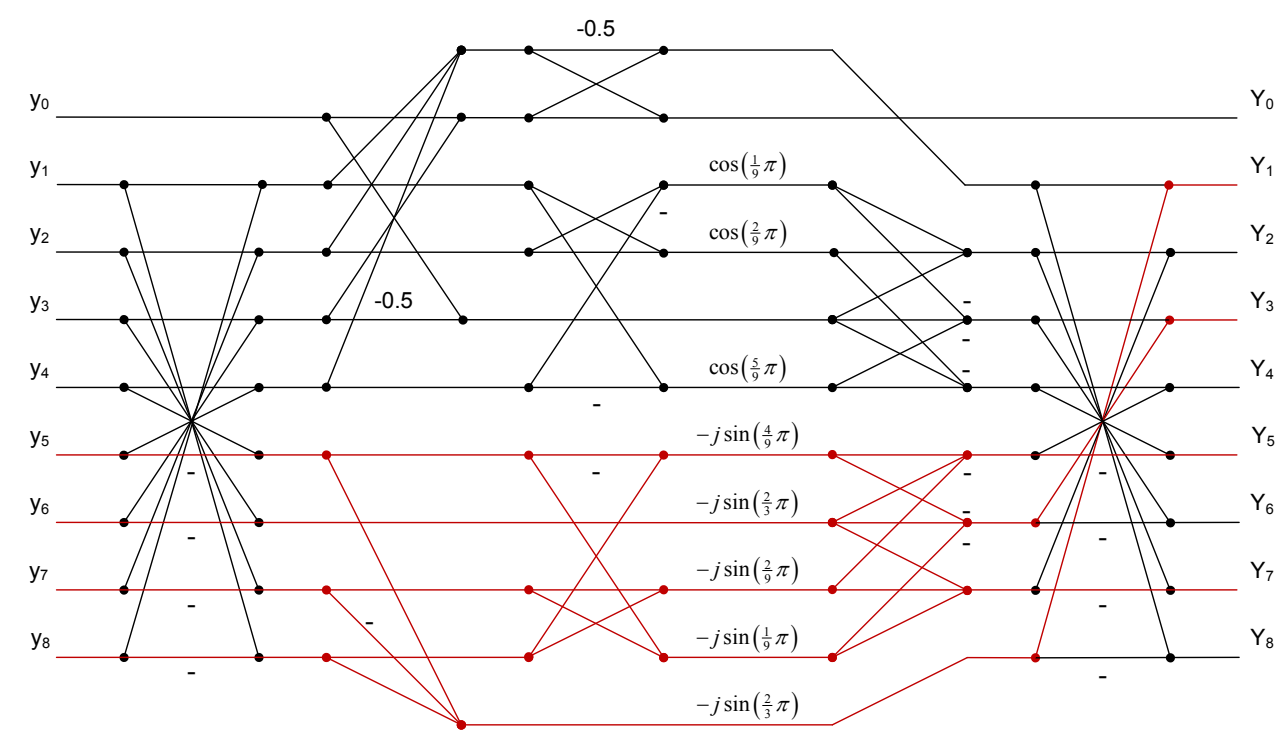

Figure 2. Flow-graph of Winograd's factorization of DFT of length 9. Paths that are needed for computation of DST-VII are shown in red.

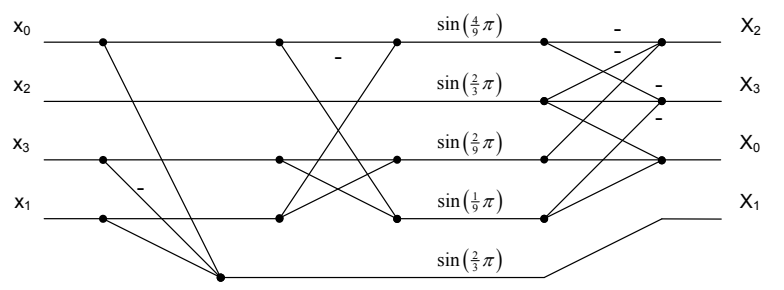

Figure 3. Flow-graph of fast factorization of DST-VII of length 4.

This produces the flow-graph for DST-VII. By reversing the direction we obtain flow-graph for DST-VI.

We now show how these steps can be executed for construction of fast transforms of length $\mathrm{N}=4$. We start with mapping (13), establishing connection between DST-VII of length 4 and 9-point DFT. Then, we pick fast factorization of DFT of length 9. In this case, we use Winograd's DFT module of length 9 described in. ${ }^{24,27}$ We show flow-graph of this algorithm in Figure 4. We use red color to show paths that are needed for computation of DST-VII. It can be easily observed that the remaining paths are irrelevant because they either receive zero input, or lead to real portion of DFT's output. Final flow-graph for computing DST-VII is show in Figure 4. Based on Figure 4 we can see that DST-VII of length 4 can be computed by using only 5 multiplications and 11 additions. Same complexity is required for computing DST-VI of length 4.

Same steps can also be repeated for construction of fast transforms of length $\mathrm{N}=8$. In this case, we can use 17-point Winograd DFT module described in. ${ }^{27,28}$ We show the final flow-graph of length-8 DST-VII in Figure 4.

Table 1. Complexity of computing DCT and DST transforms of even and odd types.

\begin{tabular}{|cccc|}
\hline \hline$N$ & DST-VI/VII & DCT-II/III & matrix-vector product \\
\hline 4 & 5 muls, 11 adds & 4 muls, 9 adds & 16 muls, 12 adds \\
8 & 21 muls, 77 adds & 12 muls, 29 adds & 64 muls, 56 adds \\
\hline
\end{tabular}




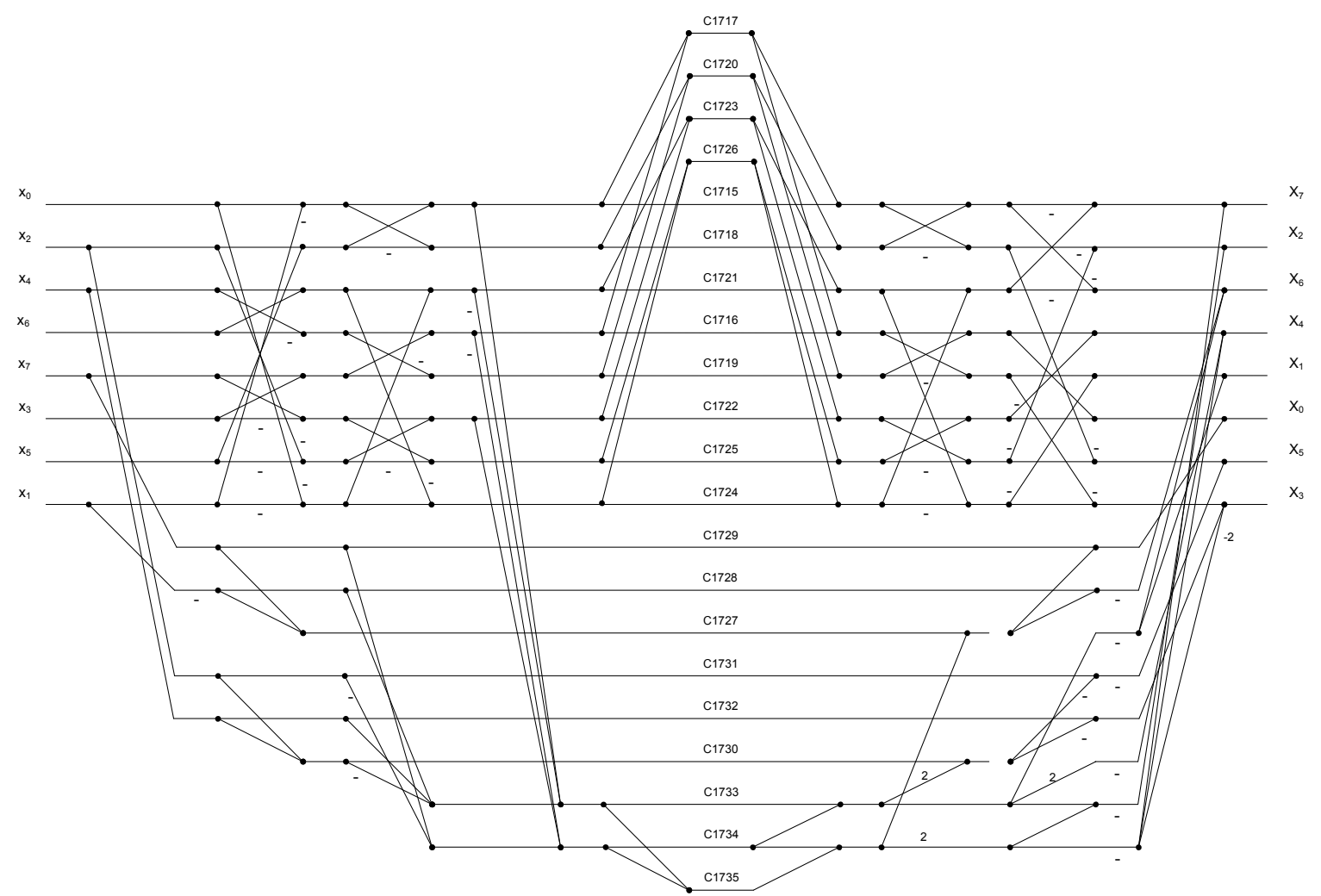

Figure 4. Flow-graph of fast factorization of DST-VII of length 8 . Factors $C 1715-C 1735$ correspond to constants appearing in length $N=17$ Winograd DFT factorization described in. ${ }^{28}$

\section{COMPLEXITY ANALYSIS}

We summarize complexity results for derived factorizations of DST-VI/VII transforms in Table 1. For comparison, we also include complexity numbers for the well-known Loeffler-Ligtenberg-Moschytz (LLM) ${ }^{12}$ factorization of DCT-II, as well as the number of operations required for straightforward matrix-vector-product computation of such transforms.

It can be observed, that our proposed DST-IV/VII factorization of length $N=4$ is very close to complexity of LLM factorization of DCT-II/III. At the same time, our proposed DST-IV/VII factorization of length $N=8$ appears to be about twice more complex than LLM factorization of DCT-II/III. ${ }^{12}$ Still, our proposed DSTIV/VII factorization of length $N=8$ offers significant (about a factor of 3 ) reduction in multiplicative complexity compared to direct computation of such transforms.

\section{REFERENCES}

[1] Rao, K. R. and Yip, P., [Discrete Cosine Transform: Algorithms, Advantages, Applications], Academic Press, Boston, MA (1990).

[2] Farrelle, P. and Jain, A., "Recursive block coding - a new approach to transform coding," IEEE Trans. Communications 34(2), 161-179 (1986).

[3] Malvar, H. S., [Signal Processing with Lapped Transforms], Artech House, Boston (1992).

[4] Chivukula, R. K. and Reznik, Y. A., "Efficient implementation of a class of MDCT/IMDCT filterbanks for speech and audio coding applications," in [IEEE Int. Conf. Acoust., Speech, Signal Processing (ICASSP)], pp. 213-216 (July 2008).

[5] Jain, A. K., "A sinusoidal family of unitary transforms," IEEE Trans. Pattern Analysis and Machine Intelligence PAMI-1, 356 -365 (Oct. 1979). 
[6] Wang, Z. and Hunt, B. R., "The discrete W transform," Applied Mathematics and Computation 16(1), 19 -48 (1985).

[7] Martucci, S. A., "Symmetric convolution and the Discrete Sine and Cosine Transforms," IEEE Trans. Signal Processing SP-42, 1038-1051 (1994).

[8] Britanak, V., Rao, K. R., and Yip, P., [Discrete Cosine and Sine Transforms: General Properties, Fast Algorithms and Integer Approximations], Academic Press - Elsevier, Oxford, UK (2007).

[9] Heideman, M. T., "Computation of an odd-length DCT from a real-valued DFT of the same length," IEEE Trans. Signal Processing 40(1), 54-61 (1992).

[10] Kok, C. W., "Fast algorithm for computing Discrete Cosine Transform," IEEE Trans. Signal Processing 45(3), 757-760 (1997).

[11] Feig, E. and Winograd, S., "On the multiplicative complexity of Discrete Cosine Transforms (Corresp.)," IEEE Trans. Information Theory IT-38, 1387-1391 (1992).

[12] Loeffler, C., Ligtenberg, A., and Moschytz, G., "Practical fast 1-D DCT algorithms with 11 multiplications," in [IEEE Int. Conf. Acoust., Speech, Signal Processing (ICASSP)], 2, 988-991 (May 1989).

[13] Reznik, Y. A. and Chivukula, R. K., "Design of fast transforms for high-resolution image and video coding," in [Applications of Digital Image Processing XXXII], Tescher, A. G., ed., Proc. SPIE 7443, 744312-1-18 (2009).

[14] R. Joshi, Y. R. and Karczewicz, M., "Efficient large size transforms for high-performance video coding," in [Applications of Digital Image Processing XXXIII], Tescher, A. G., ed., Proc. SPIE 7798, 779831-1-7 (August 2010).

[15] Sullivan, G. J., "Standardization of IDCT approximation behavior for video compression: the history and the new MPEG-C parts 1 and 2 standards," in [Applications of Digital Image Processing XXX], Tescher, A. G., ed., Proc. SPIE 6696, 669603-1-14 (August 2007).

[16] Reznik, Y. A., Hinds, A. T., Yu, L., Ni, Z., and Zhang, C.-X., "Efficient fixed-point approximations of the 8x8 Inverse Discrete Cosine Transform," in [Applications of Digital Image Processing XXX], Tescher, A. G., ed., Proc. SPIE 6696, 669617-1-17 (August 2007).

[17] Han, J., Saxena, A., and Rose, K., "Towards jointly optimal spatial prediction and adaptive transform in video/image coding," in [IEEE Int. Conf. Acoust., Speech, Signal Processing (ICASSP)], 726-729 (March 2010).

[18] Karczewicz, M., Chen, P., Joshi, R., Wang, X., Chien, W.-J., Panchal, R., Coban, M., Chong, I.-S., and Reznik, Y. A., "A hybrid video codec based on extended block sizes, recursive integer transforms, improved interpolation and flexible motion representation," in [Visual Information Processing and Communication II], Said, A., Guleryuz, O. G., and Stevenson, R. L., eds., Proc. SPIE 7882, 788203-1-15 (January 2011).

[19] Saxena, A. and Fernandes, F., "CE7: Mode-dependent DCT/DST for intra prediction in video coding," commitee input document JCTVC-D033, ISO/IEC/ITU-T Joint Collaborative Team on Video Coding, Daegu, Korea (January 2011).

[20] Bross, B., Han, W.-J., Sullivan, G. S., Ohm, J.-R., and Wiegand, T., eds., [WD4: Working Draft 4 of High-Efficiency Video Coding], committee document JCTVC-F803, ISO/IEC/ITU-T Joint Collaborative Team on Video Coding, Turin, Italy (July 2011).

[21] McCann, K., Bross, B., Han, W.-J., Sekiguchi, S., and Sullivan, G. S., eds., [HM4: HEVC Test Model 4 Encoder Description], committee document JCTVC-F802, ISO/IEC/ITU-T Joint Collaborative Team on Video Coding, Turin, Italy (July 2011).

[22] Nussbaumer, H. J., [Fast Fourier Transform and Convolution Algorithms], Springer (1981).

[23] Burrus, C. and Parks, T., [DFT/FFT and Convolution Algorithms Theory and Implementation], Wiley, New York (1985).

[24] Winograd, S., "On computing the Discrete Fourier Transform," Mathematics of Computation 32, 175-199 (January 1978).

[25] Silverman, H. F., "An introduction to programming the Winograd Fourier Transform Algorithm (WFTA)," IEEE Trans. Acoust., Speech, Signal Processing ASSP-25, 152 - 165 (April 1977).

[26] Kolba, D. and Parks, T., "A prime factor FFT algorithm using high-speed convolution," IEEE Trans. Acoust., Speech, Signal Processing ASSP-25, 281 - 294 (August 1977). 
[27] Burrus, C., [Appendix 4, Programs for Short FFTs], Connexions, available online at: http://cnx.org/content/m17646/1.4/ (September 2009).

[28] Burrus, C. and Reznik, Y. A., [ $N=17$ Winograd FFT module in $C]$, Connexions, available online at: http://cnx.org/content/m40959/1.1/ (August 2011). 